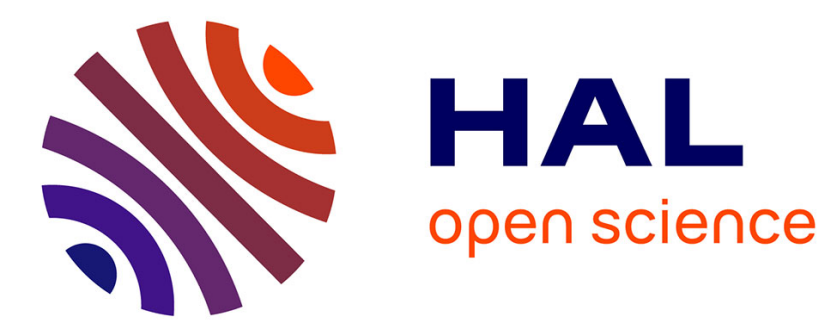

\title{
Graphene-metal interface: Two-terminal resistance of low-mobility graphene in high magnetic fields
}

V. Krstic, D. Obergfell, S. Hansel, Rikken G.L.J.A., J. Blokland, M. Ferreira, S. Roth

\section{- To cite this version:}

V. Krstic, D. Obergfell, S. Hansel, Rikken G.L.J.A., J. Blokland, et al.. Graphene-metal interface: Two-terminal resistance of low-mobility graphene in high magnetic fields. Nano Letters, 2008, 8 (6), pp.1700-1703. 10.1021/nl080634k . hal-00355407

\section{HAL Id: hal-00355407 https://hal.science/hal-00355407}

Submitted on 26 Mar 2021

HAL is a multi-disciplinary open access archive for the deposit and dissemination of scientific research documents, whether they are published or not. The documents may come from teaching and research institutions in France or abroad, or from public or private research centers.
L'archive ouverte pluridisciplinaire HAL, est destinée au dépôt et à la diffusion de documents scientifiques de niveau recherche, publiés ou non, émanant des établissements d'enseignement et de recherche français ou étrangers, des laboratoires publics ou privés. 


\title{
Graphene-Metal Interface: Two- Terminal Resistance of Low-Mobility Graphene in High Magnetic Fields
}

\author{
Vojislav Krstić ${ }^{*}{ }^{\dagger}$ Dirk Obergfell, ${ }^{\dagger}$ Stefan Hansel, ${ }^{\S}$ Geert L. J. A. Rikken, ${ }^{\S}$ Janneke H. Blokland, \\ Mauro S. Ferreira, ${ }^{\dagger}$ and Siegmar Roth:
}

Centre for Research of Adaptive Nanostructures and Nanodevices, School of Physics, Trinity College Dublin, Dublin 2, Ireland, Max-Planck-Institut für Festkörperforschung, Heisenbergstr. 1, D-70569 Stuttgart, Germany, Laboratoire National des Champs Magnétiques Pulsés, 143, Ave. de Rangueil, F-31400 Toulouse, France, and High Field Magnet Laboratory, Institute of Molecules and Materials, Radboud University Nijmegen, Toernooiveld 7, 6525 ED Nijmegen, The Netherlands

\begin{abstract}
The two-terminal magnetotransport of a single graphene layer was investigated up to a field of 55 T. The dependence of the electron transmission probability at the organo-metallic interface between the graphene and the metal electrodes was studied as a function of filling factor and electron density. A resistance-plateau spanning several tens of tesla width was observed. We argue that this plateau originates from an augmented sublattice spin-splitting due to the high surface-impurity concentration of the graphene layer. At electron densities close to the Dirac point, fingerprints of a thermally activated energy gap were observed.
\end{abstract}

The observation of a half-integer filling factor $v$ in the Quantum-Hall regime for graphene has attracted much recent interest within the scientific community. This is attributed to the possibility of describing the electrons in graphene as relativistic particles. ${ }^{1-3}$ An intriguing question arises when noting several fundamental quality differences between twodimensional electron gases (2DEGs) realized by inorganic semiconductor structures, for example, AlGaAs heterostructures and graphene when contacted to metallic electrodes. In contrast to inorganic semiconductors, graphene together with metallic electrodes represents an organo-metallic device where the interfaces are significantly more crucial $^{4-6}$ than, for example, heterostructure 2DEGs. For inorganic heterostructures, the electronic coupling to the 2DEG can be made ohmic, with transmission probabilities close to unity which remain impervious to magnetic fields. As a consequence, the Quantum-Hall plateaus can be observed with an accuracy (offset by the electrode leads) comparable to those in the Hall resistance. ${ }^{7-9}$

* Corresponding author. E-mail: krsticv@ tcd.ie. Telephone: + 3531896 3026. Fax: +35318963037.

$\dagger$ Trinity College Dublin.

\$ Max-Planck-Institut für Festkörperforschung.

$\S$ Laboratoire National des Champs Magnétiques Pulsés.

"Radboud University Nijmegen.
In case of graphene, the nature of the contact is not obvious because of the generally complex interaction between organic materials and metals. Moreover, graphene is more susceptible to surface impurities. In principle, these can be removed through heating in vacuum ${ }^{1-3}$ or in an argon/hydrogen atmosphere $^{10}$ or by driving a high current ${ }^{11}$ through the graphene under vacuum. However, the absorption of impurities is implicit to the operation of graphene devices in realistic ambient environments envisioned for future two-dimensional molecular electronics. It therefore can not be avoided as in the case of more idealized experiments. ${ }^{1-3,10,11}$ In addition, realistic graphene-device architectures may comprise invasive components as for example top-gates ${ }^{12-14}$ besides the crucial graphene/electrode interaction. In the present communication, we address the questions of how the transmission probability between graphene and metal electrode changes in the Quantum-Hall regime depending on the filling factor $v$ and the two-dimensional electron density $n_{2 \mathrm{D}}$, and thereby probe if the high surface impurity density is of any impact. Electron densities distant from and close to the Dirac point were investigated.

For our experiments, a single graphene layer (about $9 \times$ $11 \mu \mathrm{m}^{2}$ ) was deposited by the established method of micromechanical cleavage $e^{1,15}$ on a highly doped silicon wafer 


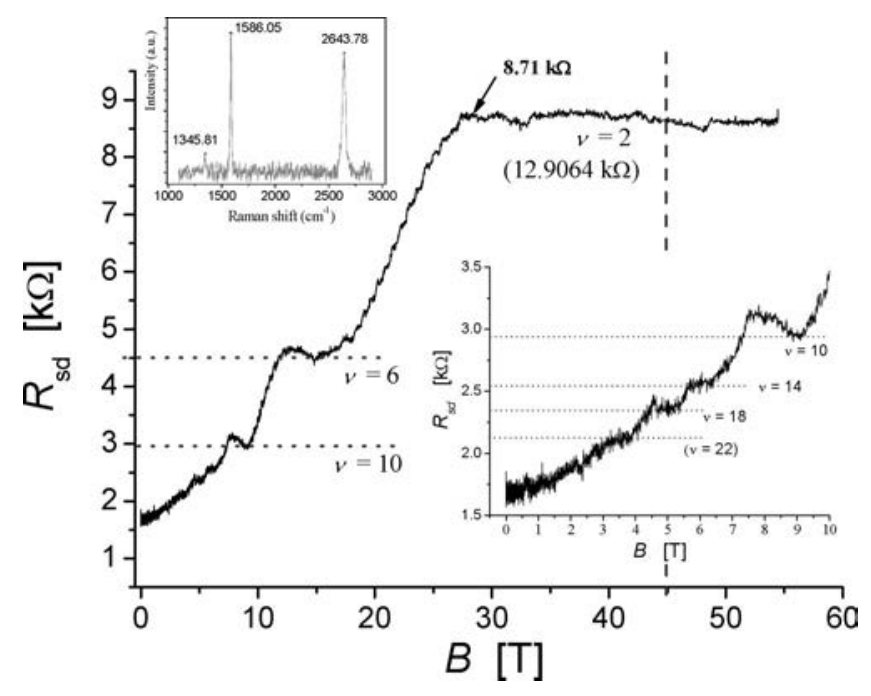

Figure 1. Upper inset: Raman spectrum of the investigated graphene layer. The position and the shape of the peaks indicate a single graphene layer. The two-terminal resistance $R_{s d}$ of a single graphene monolayer up to $55 \mathrm{~T}$ at $n_{2 \mathrm{D}} \approx 2.13 \times 10^{12} / \mathrm{cm}^{2}$. Clearly plateaus in the resistance are observed which are identified to the filling factors $6,10,14$, and 18 . The filling factor 22 is not clearly resolved (lower inset). All resistances are very close to the expected Quantum-Hall resistance $R_{Q H}$. The plateau with onset at about 25 $\mathrm{T}$ has a resistance close to $h / 3 e^{2}$. It has an extraordinary width of several tens of tesla. The grey dashed line indicates where $v=2$ plateau would have been expected (in brackets the corresponding $R_{Q H}$ value).

(300 nm silicon dioxide as dielectric) and then electrically contacted by electron-beam lithography with gold electrodes (width $\approx 14 \mu \mathrm{m}$, height $\approx 30 \mathrm{~nm}$ ). The two-terminal resistance $R_{s d}$ of the graphene layer was measured at $5 \mathrm{~K}$ in (pulsed) magnetic fields up to $55 \mathrm{~T}$.

In the upper left inset of Figure 1, a Raman spectrum of the measured graphene layer is shown which indicates that this is a single layer. The electron mobility was determined to be about $2100 \mathrm{~cm}^{2} / \mathrm{Vs}$ by gate-dependent measurements in zero magnetic field, which is significantly lower than values of the typical $10^{4} \mathrm{~cm}^{2} / \mathrm{Vs}$ or higher for QuantumHall measurements on single graphene layers reported in literature. ${ }^{1,2,16}$ This low mobility value corresponds to the circumstance that our sample was not post-treated prior to the measurements; that is, it was not in-situ heated or evacuated for removal of surface impurities like water, oxygen, and hydrocarbons, and thus exhibits a comparably high density of surface impurities.

The main graph in Figure 1 shows $R_{s d}$ at $+45 \mathrm{~V}$ which is $35 \mathrm{~V}$ above the Dirac point of the investigated layer. From geometrical considerations, this corresponds to $n_{2 \mathrm{D}} \approx 2.13$ $\times 10^{12} / \mathrm{cm}^{2}$.

Clearly, several plateaus (see also lower inset for fields smaller than $10 \mathrm{~T}$ ) can be seen which can be attributed to $v$ $=6,10,14,18$ and signatures of $v=22$. The filling factors were identified by comparison with the generally valid Landau-fan (see inset Figure 3) and match with our Raman measurements which indicated that we have a single graphene layer. ${ }^{17-19}$ The wide plateau at $R_{s d} \approx 8.71 \mathrm{k} \Omega$ will be discussed later in this communication.

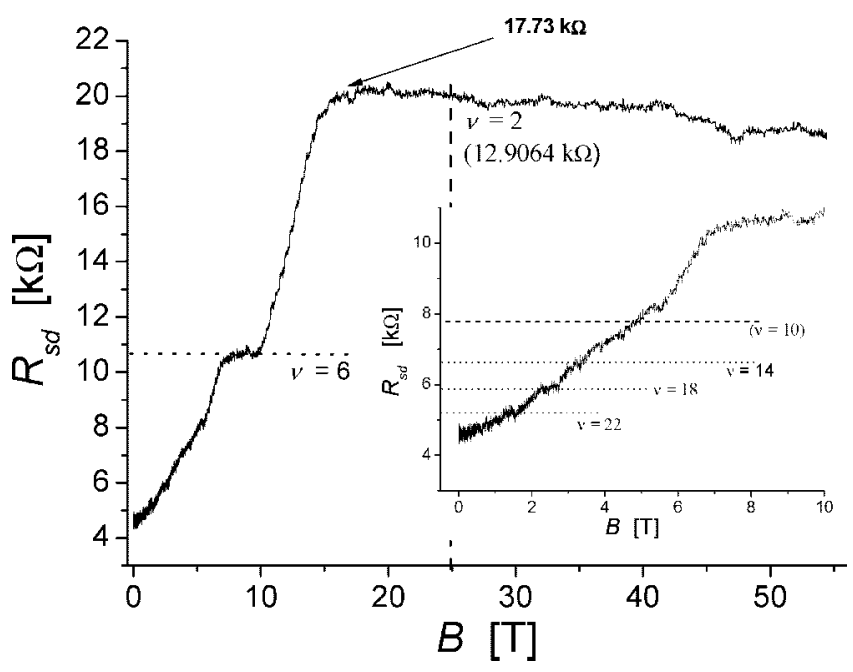

Figure 2. Two-terminal resistance $R_{s d}$ at $n_{2 \mathrm{D}} \approx 1.22 \times 10^{12} / \mathrm{cm}^{2}$ up to $55 \mathrm{~T}$. Resistance plateaus are clearly observed; however, the resistance value deviates significantly from the expected QuantumHall resistance $R_{Q H}$. A plateau with an onset at about $17.4 \mathrm{~T}$ and width of roughly $40 \mathrm{~T}$ is observed. The dashed line corresponds to the onset magnetic field for the $v=2$ plateau which is not appearing ( $R_{Q H}$ value in brackets). Inset: $R_{s d}$ up to $10 \mathrm{~T}$, showing filling factors larger than 6 . The filling factor 10 is not resolved (in brackets).

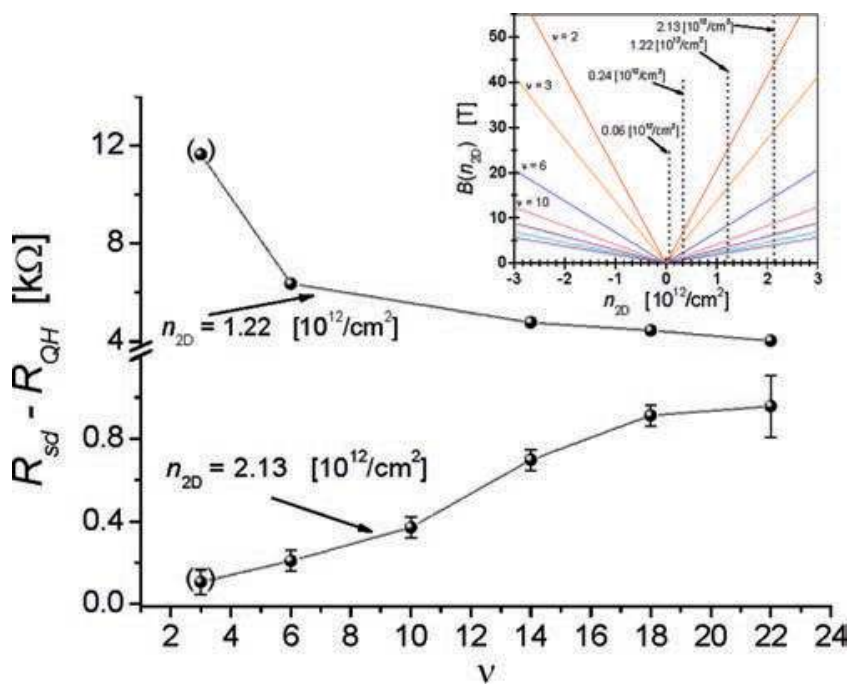

Figure 3. Difference $R_{s d}-R_{Q H}$ as a function of filling factor $v$ and for two electron densities. The difference equals the contact resistance and the constant lead resistance. Thus, $R_{s d}-R_{Q H}$ is inversely proportional to the total electron transmission probability for the graphene layer and the metallic electrodes. For the higher electron concentration, the transmission probability increases with decreasing filling factor. In contrast, for the lower electron density, the transmission probability decreases towards smaller $\nu$. The filling factor $v=3$ (brackets), belonging to the two wide plateaus, follows in both cases the trend. Inset: The generally valid Landau-fan based on theoretical considerations. Labeling of filling factors larger than 10 are omitted for simplicity. The four electron densities at which the measurements have been performed are indicated by dotted lines. The crossing of a dotted line with one of the lines of the Landau-fan defines, unambiguously, at which magnetic field which resistance plateau (filling factor) is observed.

In Figure $2, R_{s d}$ at $n_{2 \mathrm{D}} \approx 1.22 \times 10^{12} / \mathrm{cm}^{2}$ up to $55 \mathrm{~T}$ is shown. In the lower inset, measured data below $8 \mathrm{~T}$ is plotted. The filling factors which could be identified were $v=6$, $14,18,22$. The filling factor 10 could not be resolved clearly. 
Similar to the measurements at higher $n_{2 \mathrm{D}}$, again a wide plateau appears $\left(R_{s d}=17.73 \mathrm{k} \Omega\right)$.

The first striking difference to be observed is that for $n_{2 \mathrm{D}}$ $\approx 1.22 \times 10^{12} / \mathrm{cm}^{2}$ the plateau-resistances are, in all cases, significantly higher than those for $n_{2 \mathrm{D}} \approx 2.13 \times 10^{12} / \mathrm{cm}^{2}$.

In order to extract information on the total transmission probability, $R_{s d}-R_{Q H}$ is plotted against the filling factor $v$ (Figure 3 ) for the two $n_{2 \mathrm{D}} . R_{s d}$ is the sum of contact resistances, the Quantum-Hall resistance $R_{Q H}$ and the lead resistance. The lead resistance is constant and was measured to be about $200 \Omega$ at room temperature on (short-circuited) identical electrode layout samples without graphene. Thus, $R_{s d}-R_{Q H}$ is directly proportional to the contact resistances each being inversely proportional to the graphene/metal electron transmission probability.

For both electron densities, a significant dependence of the inverse total transmission probability on the filling factor $v$ is apparent. Moreover, the dependence is opposite for the two electron densities, and the general transmission probability increases with higher $n_{2 \mathrm{D}}$. Note, in particular, at higher $n_{2 \mathrm{D}}$, the contact resistance plus the lead resistance are in the range of only a few hundred ohms which indicates a transmission-probability range close to unity for this electron density.

Therefore, it can be concluded that the transmission probability of electrons between graphene and metal electrodes is highly dependent on both filling factor and twodimensional electron density reflecting the complex nature of an organo-metallic interface.

We now turn to the discussion of the wide plateaus observed in Figure 1 and Figure 2. The onset of the two plateaus is at the magnetic fields 17.4 and $28 \mathrm{~T}$ for $1.22 \times$ $10^{12} / \mathrm{cm}^{2}$ and $2.13 \times 10^{12} / \mathrm{cm}^{2}$, respectively. This is wellbelow those fields expected for $v=2$ (about 25 and $44 \mathrm{~T}$ ). A possible explanation could be the spin- and the sublattice degeneracy splitting of Landau-levels (LLs), which was recently reported in high magnetic fields up to $45 \mathrm{~T}$ in a high-mobility sample. ${ }^{20}$ However, there, the LL sublattice splitting was only observed clearly for the LL $n=0 .{ }^{20} \mathrm{In}$ the data in Figure 1 and 2, the onset of the two long plateaus does not coincide with $v=4$ which would be a consequence of an exclusive spin-splitting of the $n=1$ LL (c.f., ref 20). Moreover, the long plateau at $n_{2 \mathrm{D}} \approx 2.13 \times 10^{12} / \mathrm{cm}^{2}$ has a value of about $8.71 \mathrm{k} \Omega$ for $R_{s d}$. This is very close to $h / 3 e^{2}$ $=8.60 \mathrm{k} \Omega$, that is, the $R_{Q H}$ for the filling factor $v=3$, and falls in the transmission-probability trend with high transmission probabilities for all filling factors (Figure 3). Assuming that sublattice degeneracy is lifted at low fields ${ }^{20}(<9 \mathrm{~T})$ and plotting the $n_{2 \mathrm{D}}$ dependence of a $v=3$ plateau in the Landau-fan, we find for $1.22 \times 10^{12} / \mathrm{cm}^{2}$ and $2.13 \times 10^{12} /$ $\mathrm{cm}^{2}$ the expected position of this plateau to be at about 16.9 and $28 \mathrm{~T}$, respectively. This coincides remarkably well with our experimentally observed onset of the two wide plateaus. The low-field sublattice splitting may have occurred in our graphene layer because of the surface impurities (the reason of the low mobility) introducing some anisotropic potential. The plateau width matches with the square-root dependence of the LL energy $\left.E_{n}=\sqrt{ } 2 e \hbar v_{F}^{2}|n| B\right\}$ for the magnetic field

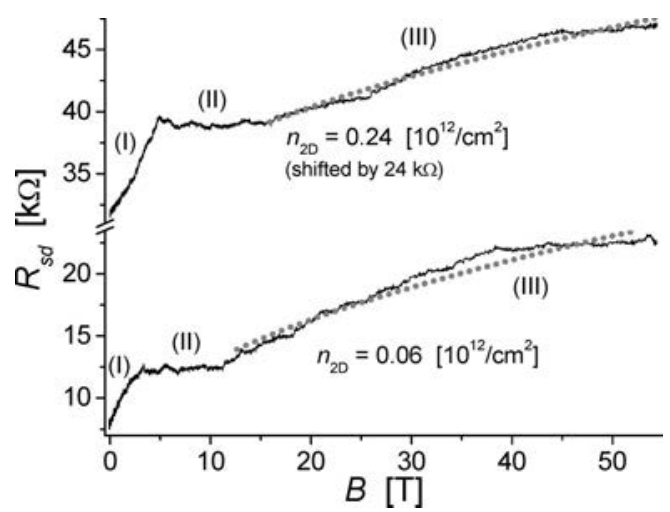

Figure 4. $R_{s d}$ vs magnetic field for two electron densities close to the Dirac point. The upper curve is shifted upward by $24 \mathrm{k} \Omega$ for clarity. In both cases, first a phase of strong increase in resistance (I) is observed, followed by a plateau-like phase (II), and then by a nonlinear increase (III) with magnetic field strength. The plateaulike phases can not be attributed to a particular filling factor. The nonlinear increase is suggested to originate from a thermally activated energy gap. The dotted lines are fits following the recently pointed out assumption that this gap is of excitonic nature and increases with $\sqrt{ } B{ }^{20,21}$ However, the origins of the plateau-like feature remain unsolved within this model.

( $v_{F}$ is the Fermi velocity; $B$ is the magnetic-field strength, $n$ $=0, \pm 1, \pm 2$,$) . That is, at higher magnetic fields, a$ saturation effect should be observed in $R_{s d}$. However, the $v$ $=4$ plateau absence remains unexplained, possibly resolvable if the magnetic-field dependence of the sublattice-splitting energy is significantly higher than the spin-splitting energy of less than a few millielectronvolts ${ }^{20}$ for our low-mobility sample. A closer theoretical and experimental investigation of this phenomenon is without doubt justified and will be addressed in more detail elsewhere.

Finally, we turn to electron densities close to the Dirac point. The $R_{s d}$ up to $55 \mathrm{~T}$ are shown in Figure 4 for $0.06 \times$ $10^{12} / \mathrm{cm}^{2}$ and $0.24 \times 10^{12} / \mathrm{cm}^{2}$ (upper curve shifted by 24 $\mathrm{k} \Omega$ for clarity). In both cases, there is a strong increase in the resistance within a few tesla $(<6 \mathrm{~T})$ which develops into a plateau-like phase. At higher fields, $R_{s d}$ increases in a nonlinear way, with indications for a saturation, and there is no significant difference in the overall resistance values for the two electron concentrations. The positions of the plateau-like phases do not coincide with any filling factor on the basis of the Landau-fan. However, this phase must be governed by the $n=0 \mathrm{LL}$, since at these low electron densities the Quantum-Hall plateaus are very dense and all filling factors are already passed through up to $7 \mathrm{~T}$ (c.f., inset Figure 3).

Regarding the smooth nonlinear $R_{s d}$ increase with magnetic field, it was reported ${ }^{20}$ that, at electron densities close to the Dirac point and fields larger than about $10 \mathrm{~T}$, an intriguing increase in the longitudinal resistance develops. This coincides with the observation of an energy gap opening at $v=$ 0 of unknown origin. ${ }^{20}$ The strong increase of $R_{s d}$ within a few tesla in Figure 4 matches with the longitudinal resistance increase observed in ref 20 . This suggests that the measured magnetic-field dependence of $R_{s d}$ for these electron densities is a qualitative fingerprint of the energy gap. The gap is 
predicted to be of excitonic nature and to increase with $\sqrt{ } B$, according to recent theoretical considerations. ${ }^{21}$ This gap should be thermally activated and may contribute in first order a component proportional to $\exp \left(\sqrt{ } B / k_{\mathrm{B}} T\right)$ to $R_{s d}$. Fits on both curves in the region after the plateau-like phase using $R_{s d} \propto \exp \left(\sqrt{ } B / k_{\mathrm{B}} T\right)$ were performed (gray dotted line). The match is reasonable; however, the existence of the plateaulike phase indicates that the theory does not fully account for the observed experimental data.

In summary, we have carried out two-terminal measurements up to $55 \mathrm{~T}$ on a single graphene layer with high surface-impurity concentration. In this low-mobility graphene layer, at higher electron densities, clear plateaus in the twoterminal resistance are observed which could be attributed to different filling factors.

It was demonstrated that the transmission probability for electrons between graphene and metal electrodes is not constant and depends, in a complex way, on the filling factor and the two-dimensional electron density. A plateau with all characteristics of the filling factor 3 was observed, which shows an unusual width of several tens of tesla, suggesting that the surface impurities of the graphene may have led to a low-field lift of the sublattice degeneracy. At electron densities close to the Dirac point, the resistance provides indications on a theoretically predicted magnetic-field induced excitonic gap. However, the theory does not fully explain all of the features we observe.

Acknowledgment. The authors are grateful for helpful discussions with K. von Klitzing and J. Smet on the Quantum-Hall resistance plateau's development in high magnetic fields. The authors also thank V. Skakalova for support in Raman spectroscopy and G. Cross and W. McKenzie for careful reading of the manuscript and valuable comments. Part of this work has been supported by EuroMagNET under the EU Contract No. RII3-CT-2004-506239. M.S.F. acknowledges financial support from Science Foundation Ireland.

\section{References}

(1) Novoselov, K. S.; Geim, A. K.; Morozov, S. V.; Jiang, D.; Katsnelson, M. I.; Grigorieva, I. V.; Dubonos, S. V.; Firsov, A. A. Nature 2005, 438, 197.

(2) Zhang, Y.; Tan, Y. W.; Stormer, H. L.; Kim, P. Nature 2005, 438, 201

(3) Gusynin, V. P.; Sharapov, S. G. Phys. Rev. Lett. 2005, 95, 146801.

(4) Wang, W; Lee, T.; Kamdar, M.; Reed, M. A.; Stewart, M. P.; Hwang, J. J.; Tour, J. M. Ann. N.Y. Acad. Sci. 2003, 1006, 36.

(5) Davis, J. J.; Morgan, D. A.; Wrathmell, C. L.; Axford, D. N.; Zhao, J.; Wang, N. J. Mater. Chem. 2005, 15, 2160.

(6) Sanvito, S. Nat. Mat. 2007, 6, 803.

(7) Fang, F. F.; Stiles, P. J. Phys. Rev. B 1983, 27, 6487.

(8) Fang, F. F.; Stiles, P. J. Phys. Rev. B 1984, 29, 3749.

(9) Rikken, G. L. J. A.; van Haaren, J. A. M. M.; van der Wel, W.; van Gelder, A. P.; van Kempen, A. P.; Wyder, P.; André, J. P.; Ploog, K.; Weimann, G. Phys. Rev. B 1988, 37, 6181.

(10) Ishigami, M.; Chen, J. H.; Cullen, W. G.; Fuhrer, M. S.; Williams, E. D. Nano Lett. 2007, 7, 1643.

(11) Moser, J.; Barreiro, A.; Bachtold, A. Appl. Phys. Lett. 2007, 91, 163513.

(12) Lemme, M. C.; Echtermeyer, T. J.; Baus, M.; Kurz, H. IEEE Electronic Device Letter 2007, 28, 282.

(13) Huard, B.; Sulpizino, J. A.; Standfer, N.; Todd, K.; Yang, B.; Goldhaber-Gordon, D. H. Phys. Rev. Lett. 2007, 98, 236803.

(14) Williams, J. R.; DiCarlo, L.; Marcus, C. M. Science 2007, 317, 638.

(15) Novoselov, K. S.; Jiang, D.; Shedin, F.; Booth, T. J.; Khotkevich, V. V.; Morozov, S. V.; Geim, A. K. Proc. Natl. Acad. Sci. U.S.A. 2005, 102, 10451.

(16) Novoselov, K. S.; Jiang, Z.; Zhang, Y.; Morozov, S. V.; Stormer, H. L.; Zeitler, U.; Maan, J. C.; Boebinger, B. S.; Kim, P.; Geim, A. K. Science 2007, 315, 1379.

(17) Casiraghi, C.; Hartschuh, A.; Lidorikis, E.; Qian, H.; Harutyunyan, H.; Gokus, T.; Novoselov, K. S.; Ferrari, A. C. Nano Lett. 2007, 7, 2711.

(18) Ferrari, A. C.; Meyer, J. C.; Scardaci, V.; Casiraghi, C.; Lazzeri, M.; Mauri, F.; Piscanec, S.; Jiang, D.; Novoselov, K. S.; Roth, S.; Geim, A. K. Phys. Rev. Lett. 2006, 97, 187401.

(19) Haluǩa, M.; Obergfell, D.; Meyer, J. C.; Scalia, G.; Ulbricht, G.; Krauss, B.; Chae, D. H.; Lohmann, T.; Lebert, M.; Kaempgen, M.; Hulman, M.; Smet, J.; Roth, S.; von Klitzing, K. Phys. Status Solidi B 2007, 244, 4143.

(20) Zhang, Y.; Jiang, Z.; Small, J. P.; Purewal, M. S.; Tan, Y. W.; Fazlollahi, M.; Chudow, J. D.; Jaszczak, J. A.; Stormer, H. L.; Kim, P. Phys. Rev. Lett. 2006, 96, 136806.

(21) Khveshchenko, D. V. Phys. Rev. Lett. 2001, 87, 206401. NL080634K 